\title{
Predictors for Gingival Index in Middle-Aged Asian Indians with Type 2 Diabetes from South India: A Cross-Sectional Observational Study
}

\author{
S. Jai Karthik $\mathbb{D}^{1,2}$ Shajith Anoop, ${ }^{1}$ R. Suresh Kumar, ${ }^{3}$ and M. V. Usha Rani ${ }^{1}$ \\ ${ }^{1}$ Department of Environmental Sciences, Bharathiar University, Coimbatore, Tamil Nadu, India \\ ${ }^{2}$ RVS Dental College, Coimbatore, Tamil Nadu, India \\ ${ }^{3}$ Suresh Diabetes Centre, Vadavalli, Coimbatore, Tamil Nadu, India \\ Correspondence should be addressed to S. Jai Karthik; jkperio@yahoo.com
}

Received 26 June 2017; Revised 2 November 2017; Accepted 19 November 2017; Published 2 January 2018

Academic Editor: Andreas K. Nussler

Copyright (C) 2018 S. Jai Karthik et al. This is an open access article distributed under the Creative Commons Attribution License, which permits unrestricted use, distribution, and reproduction in any medium, provided the original work is properly cited.

\begin{abstract}
Asian Indians develop type 2 diabetes mellitus (T2DM) much earlier as compared to White Caucasians, due to unique phenotypic and genetic architecture. Periodontitis in T2DM patients is often a neglected clinical feature. This study was conducted to derive predictor variables for gingival index in middle-aged Asian Indians with T2DM in a semiurban population of Dravidian ethnicity from Tamil Nadu, India. T2DM patients $(n=232$, mean age: $50.6 \pm 10.4$ years) with periodontitis $(n=123$, mean age: $54.3 \pm 2.4$ years) and without periodontitis ( $n=109$, mean age: $55.2 \pm 3.1$ years) were recruited between 2014 and 2016 by purposive sampling method. Dental examinations for pocket depth (PD) and clinical attachment level (CAL) were performed and gingival index was calculated. Fasting venous blood samples were analysed for measures of glycaemia and cholesterol. Significant positive correlation $(p<0.01)$ was observed for gingival index with glycosylated haemoglobin (HbAlc), pocket depth, presence of T2DM, and clinical attachment level. Stepwise multiple linear regression analysis derived increased pocket depth $(p<0.01)$, elevated HbAlc $(p<0.01)$, clinical attachment level $(p<0.01)$, and presence of diabetes $(p<0.01)$ as significant predictors $\left(r^{2}\right.$ value $\left.=0.67\right)$ for increased gingival index in middle aged patients with T2DM. These variables significantly $(p<0.01)$ predispose middle-aged T2DM patients to increased gingival index, thus warranting appropriate intervention.
\end{abstract}

\section{Introduction}

The incidence of type 2 diabetes mellitus (T2DM) is ever growing in Southern Asia and is predicted to increase along with urbanization [1]. Among other secondary complications $[2,3]$ a close link exists between T2DM, periodontitis, and liver diseases [4]. Importantly, periodontal disease is associated with cardiovascular diseases and is predictive of future cardiac events [5]. A bidirectional relationship exists between T2DM and periodontal disease [6] which is attributed to persisting hyperglycemia, leading to an exaggerated immunoinflammatory response to pathogenic microbial challenge in the gingiva [7]. In T2DM, persisting hyperglycemia causes non-enzymatic glycation and oxidation of proteins and lipids, leading to the subsequent formation of advanced glycation end products (AGEs) [8]. AGEs with accompanying markers for increased oxidative stress have been demonstrated in human gingiva in T2DM subjects with periodontitis, leading to rapid and severe periodontal tissue destruction [9].

Importantly, dental health in T2DM patients is indicative of glycaemic control and can also predict future risk of retinopathy and neuropathy [10]. With the increasing prevalence of T2DM in Asian Indians and its comorbidities including periodontitis, it is alarming to note that dental health in T2DM patients, especially the middle aged, is much neglected.

We hypothesized that glycaemic status in T2DM patients is closely related to gingival index and aimed to determine the clinical variables that correlate with gingival index in a sample of middle aged T2DM subjects with and without chronic periodontitis from a semiurban population of South India. We aimed to derive simple predictors that can be applied for 
early detection of periodontal risk in T2DM patients aged above 50 years, such that preventive therapy can be initiated.

\section{Methods}

This cross-sectional study was conducted in a dental care centre at Coimbatore, Tamil Nadu. Ethical approval was obtained from the Institutional Ethics Committee of RVS Dental College, Coimbatore, prior to the commencement of the study. The objectives were explained to all participating subjects and signed and written consent was obtained prior to enrollment in the study.

2.1. Subject Population and Selection. Purposive convenient sampling method was adopted. A minimum sample size of 80 subjects for each arm was determined with an alpha error of $5 \%$ at $90 \%$ power $(95 \% \mathrm{CI})$. A total of 232 middle-aged (above 50 years of age) patients with T2DM were recruited. Inclusion criteria were presence of clinically diagnosed T2DM at least 12 months prior to the start of the study and at least 4 fully erupted teeth. A questionnaire based interview was administered to participants to assess awareness of dental health and hygiene. Quality of dental hygiene was determined as frequency and method of brushing, frequency of tooth brush change, dental flossing, number of dental visits for scaling per year, habitual chewing of tobacco, and pyorrhea. Clinical history of patients was obtained with consent from the treating physician and the patient. T2DM subjects with chronic history of smoking, alcoholism, dental abscesses, overt pyorrhea, use of dental braces, and capping or missing teeth were excluded from the study.

2.2. Study Protocol. Periodontal examinations were performed using sterile instruments by a single examiner for all study subjects. Pocket depth (PD) measurements were obtained by using William's periodontal probe. The PD was measured from the free gingival margin to the base of the pocket. The probe was maintained parallel to the long axis of the tooth. Pocket depth and clinical attachment level (CAL) were measured for each tooth at 6 sites, namely, mesiobuccal, midbuccal, distobuccal, mesiolingual, midlingual, and distolingual. Clinical attachment level (CAL) was determined by measuring the distance from the cement enamel junction (CEJ) to the gingival margin with a William's periodontal probe. Gingival index (GI) was determined and recorded at 4 gingival sites per tooth using the following criteria: (0) normal gingiva, (1) mild gingivitis without bleeding on probing, (2) moderate gingivitis with bleeding on probing, and (3) severe gingivitis with ulceration and spontaneous bleeding. The sum of the scores from the four areas of each tooth was divided by 4 to derive the gingival index for that tooth. The mean GI score was obtained after calculating individual GI [11].

Fasting blood glucose (FBG) after an eight-hour overnight fast, $120 \mathrm{~min}$ postprandial blood glucose (PPBG), glycosylated hemoglobin (HbAlc), and serum cholesterol were analysed for all subjects on the same day. FBG and PPBG were analysed by glucose-oxidase peroxidase method. HbAlc was estimated by high performance liquid chromatography and expressed as percentage value. Serum lipid profile was measured by methods mentioned in a previous study from our group [12]. Overweight and obesity were defined in accordance with the cut-offs for Asian Indians [13]. Dyslipidemia was defined by the criteria formulated by the National Cholesterol Education Program, Adult Treatment Panel III (NCEP-ATP III) [14]. T2DM was diagnosed if fasting plasma glucose was $\geq 126 \mathrm{mg} / \mathrm{dL}$ and/or $120 \mathrm{~min}$ postprandial plasma glucose was $\geq 200 \mathrm{mg} / \mathrm{dL}$ or there was self-reported medication for diabetes by the participant [15].

\section{Data Analysis}

Statistical analysis was performed using statistical software STATA (version 14.2, StataCorp, College Station, Texas, USA). Data was checked for normative distribution and an independent samples $t$-test was applied for continuous variables. Pearson's correlation test and stepwise multiple linear regression (SMLR) analysis were used to analyse correlations and to derive predictor variables, respectively. The $p$ value $\leq$ 0.05 was considered as statistically significant.

\section{Results}

The sample size was matched for age and BMI. Of the 232 obese, middle aged T2DM subjects recruited in this study, 123 subjects with T2DM (53.01\%) had no indications of periodontitis whereas 109 subjects $(46.55 \%)$ with T2DM had chronic periodontitis. The mean values of BMI, FBG, HbAlc, mean pocket depth, and gingival index were comparatively higher in T2DM patients with significant differences in clinical attachment level (CAL), probing depth (PPD), and gingival index (Table 1). Genderwise comparisons revealed no significant differences in the clinical profile, clinical attachment level, gingival index, and pocket depth (Table 2).

In this study, patients with T2DM and periodontitis had periodontal pockets measuring at least $4 \mathrm{~mm}$ deep with bleeding on probing and radiographic evidence of bone loss above 50\%. T2DM patients without periodontitis had periodontal pockets measuring less than $4 \mathrm{~mm}$ deep with no radiographic evidence of bone loss. Correlation statistics revealed significant positive correlation $(p<0.001)$ between gingival index, pocket depth, presence of diabetes, and clinical attachment level (Table 3). Significant positive correlation was also observed between $\mathrm{HbAlc}$ and pocket depth (Figure 1(a)) and clinical attachment level (Figure 1(b)). Irrespective of age and gender, the predictive variables for gingival index in the study cohort were HbAlc $(p<0.01)$, pocket depth $(p<0.01)$, and presence of diabetes $(p<0.01)$, with a regression coefficient $\left(r^{2}\right)$ value of 0.67 (Table 4$)$.

Genderwise correlations revealed significant positive correlation of gingival index with age, $(p<0.001)$, BMI $(p<$ $0.001)$, HbAlc $(p<0.001)$, pocket depth $(p<0.001)$, and clinical attachment level $(p<0.001)$ in males. However, though significant correlations were observed for HbAlc, pocket depth $(p<0.001)$, and clinical attachment level $(p<$ $0.001)$, no significant correlation was observed for gingival index with age and BMI in females (Table 5). 
TABLE 1: Descriptive statistics between groups.

\begin{tabular}{|c|c|c|c|}
\hline Demographic \& clinical profile & Group $1(n=123)$ & Group $2(n=109)$ & $p$ value \\
\hline Age (years) & $54.3 \pm 2.4$ & $55.2 \pm 3.1$ & 0.28 \\
\hline Body mass index $\left(\mathrm{kg} / \mathrm{m}^{2}\right)$ & $25.9 \pm 2.5$ & $26.4 \pm 3.5$ & 0.23 \\
\hline Fasting blood glucose (mg/dl) & $126.07 \pm 45.4$ & $131.2 \pm 63.7$ & 0.47 \\
\hline $\begin{array}{l}\text { Postprandial blood glucose } \\
\text { (mg/dl) }\end{array}$ & $187.8 \pm 78.2$ & $182.34 \pm 78.5$ & 0.58 \\
\hline Glycosylated haemoglobin (\%) & $7.1 \pm 1.3$ & $7.7 \pm 1.8$ & $<0.05$ \\
\hline Total cholesterol (mg/dl) & $229.7 \pm 43.1$ & $218.28 \pm 42.3$ & $<0.05$ \\
\hline $\begin{array}{l}\text { High-density lipoprotein } \\
\text { cholesterol (mg/dl) }\end{array}$ & $40.29 \pm 8.9$ & $42.45 \pm 8.7$ & 0.06 \\
\hline $\begin{array}{l}\text { Low-density lipoprotein } \\
\text { cholesterol (mg/dl) }\end{array}$ & $146.74 \pm 34.4$ & $137.97 \pm 33.4$ & 0.05 \\
\hline Triglycerides (mg/dl) & $147.15 \pm 50.6$ & $147.66 \pm 50.0$ & 0.94 \\
\hline Clinical attachment level (mm) & $5.38 \pm 1.2$ & $7.78 \pm 1.1$ & $<0.01$ \\
\hline Pocket depth on probing $(\mathrm{mm})$ & $3.91 \pm 1.2$ & $5.79 \pm 1.4$ & $<0.01$ \\
\hline Gingival index & $1.83 \pm 0.4$ & $2.86 \pm 0.3$ & $<0.01$ \\
\hline
\end{tabular}

$p<0.05$ : statistically significant; Group 1: T2DM subjects without periodontitis; Group 2: T2DM subjects with periodontitis.

TABLE 2: Descriptive statistics across gender.

\begin{tabular}{|c|c|c|c|}
\hline Demographic \& clinical profile & Males $(n=130)$ & Females $(n=102)$ & $p$ value \\
\hline Age (years) & $50.1 \pm 10.2$ & $51.1 \pm 10.7$ & 0.48 \\
\hline Body mass index $\left(\mathrm{kg} / \mathrm{m}^{2}\right)$ & $26.1 \pm 2.3$ & $26.1 \pm 3.7$ & 0.90 \\
\hline Fasting blood glucose (mg/dl) & $127.6 \pm 50.0$ & $129.5 \pm 60.4$ & 0.78 \\
\hline Postprandial blood glucose (mg/dl) & $188.5 \pm 77.1$ & $181.1 \pm 76.8$ & 0.47 \\
\hline Glycosylated haemoglobin (\%) & $7.3 \pm 1.7$ & $7.4 \pm 1.5$ & 0.85 \\
\hline Total cholesterol (mg/dl) & $224.9 \pm 43.9$ & $223 \pm 42.6$ & 0.81 \\
\hline High-density lipoprotein cholesterol (mg/dl) & $41.9 \pm 9.0$ & $40.3 \pm 8.6$ & 0.19 \\
\hline Low-density lipoprotein cholesterol (mg/dl) & $145.6 \pm 35.2$ & $138 \pm 32.4$ & 0.13 \\
\hline Triglycerides (mg/dl) & $148.1 \pm 53.4$ & $146.4 \pm 46.1$ & 0.80 \\
\hline Clinical attachment level (mm) & $6.6 \pm 1.6$ & $6.3 \pm 1.7$ & 0.28 \\
\hline Pocket depth on probing (mm) & $4.8 \pm 1.4$ & $4.7 \pm 1.6$ & 0.90 \\
\hline Gingival index & $2.3 \pm 0.6$ & $2.4 \pm 0.6$ & 0.69 \\
\hline
\end{tabular}

$p<0.05$ : statistically significant.

TABLE 3: Correlation of clinical parameters with gingival index in the study cohort.

\begin{tabular}{lcc}
\hline Clinical variable & Correlation coefficient & $p$ value \\
\hline Body mass index $\left(\mathrm{kg} / \mathrm{m}^{2}\right)$ & 0.13 & $\mathrm{NS}$ \\
Glycosylated haemoglobin $(\%)$ & 0.28 & $<0.01$ \\
High density lipoprotein cholesterol $(\mathrm{mg} / \mathrm{dl})$ & 0.16 & $\mathrm{NS}$ \\
Presence of diabetes & 0.78 & $<0.01$ \\
Clinical attachment level $(\mathrm{mm})$ & 0.77 & $<0.01$ \\
Pocket depth $(\mathrm{mm})$ & 0.63 & $<0.01$ \\
\hline
\end{tabular}

$p<0.05$ : statistically significant; NS: not significant.

Gender based analysis for predictors of gingival index revealed pocket depth and clinical attachment level as significant determinants in males and females (Table 6).

We analysed the data for correlations on the basis of medications, namely, OADs (metformin + sulphonylureas) (Group 1) and insulin (Group 2). For T2DM patients on OADS, significant positive correlation was observed for gingival index with fasting blood glucose $(p<0.05)$, triglycerides $(p<0.01)$, pocket depth $(p<0.001)$, and clinical attachment level, but significant negative correlation was observed with age $(p<0.01)$. Interestingly, in T2DM patients on insulin therapy, significant positive correlation was observed for gingival index with HbAlc $(r=0.21$, $p<0.01)$, pocket depth $(r=0.57, p<0.001)$, and 
TABLE 4: Predictors of gingival index in patients with T2DM.

\begin{tabular}{lccc}
\hline Predictor variables & $\begin{array}{c}\text { Standardized coefficient } \\
\text { (beta value })\end{array}$ & $p$ value & $95 \%$ confidence interval \\
\hline Glycosylated haemoglobin (\%) & 0.16 & $<0.01$ & $0.007-0.058$ \\
Pocket depth $(\mathrm{mm})$ & 0.24 & $<0.01$ & $0.19-0.27$ \\
Presence of diabetes & 0.59 & $<0.01$ & $0.08-0.15$ \\
\hline
\end{tabular}

$p<0.05$ : statistically significant;regression coefficient value: $\left(r^{2}\right)=0.67$.

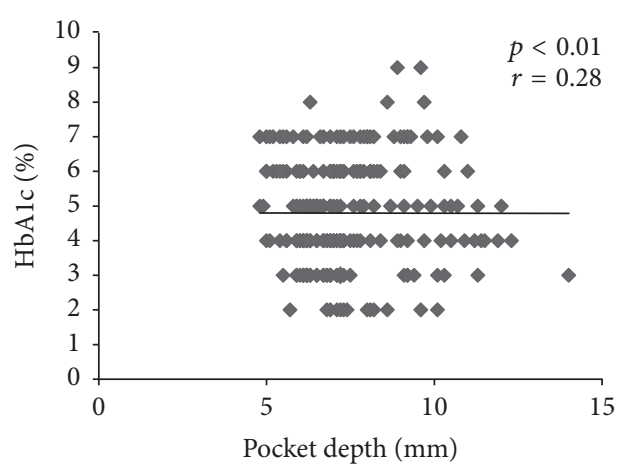

(a)

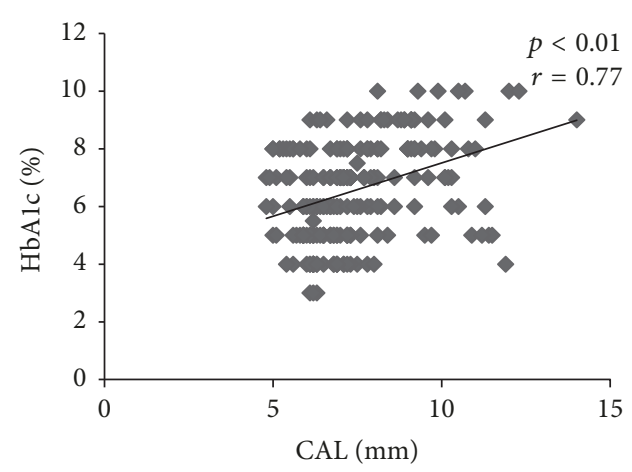

(b)

FIGURE 1: (a) Scatter plot showing correlation between HbAlc and pocket depth. (b) Scatter plot showing correlation between HbAlc and clinical attachment level (CAL).

clinical attachment level $(r=0.73, p<0.001)$ but not for fasting blood glucose and triglycerides (Table 7). Above all, SMLR analysis for predictors of gingival index based on medications did not derive gingival index, pocket depth, and clinical attachment as significant determinants.

\section{Discussion}

Systemic inflammation is significantly elevated in the presence of obesity, insulin resistance, hyperglycemia, and T2DM which derange the normal metabolic and endocrine functions of adipose tissue, resulting in increased production of fatty acids, hormones, cytokines, and acute phase reactants [16]. T2DM is a risk factor for gingivitis and periodontitis while the level of glycaemic control appears to be an important determinant in this relationship [17]. In our study, significant differences in gingival index were observed between the two groups. The increased levels of attachment loss and pocket depth in T2DM subjects with periodontitis are similar to observations from recent studies [18, 19]. Further, we observed that gingival index is positively associated with glycosylated haemoglobin despite lack of significant differences in blood glucose levels in both groups. In another case-control study, positive associations between $\mathrm{HbAlc}$ and $\mathrm{CAL}$ and BOP were observed suggesting the need for rigorous glycaemic control to alleviate periodontitis in patients with T2DM [20].

In the present study, three predictive factors for gingival index, namely, HbAlc, pocket depth, and presence of diabetes, were obtained. A combination of these factors leads to elevated risk of periodontitis upto $67 \%$ in middle aged subjects with T2DM. Genderwise comparisons revealed significant correlation of gingival index with $\mathrm{HbA1c}$, pocket depth, and CAL in males and females. Specifically, a higher degree of correlation was observed in females for pocket depth and clinical attachment. Interestingly, pocket depth, clinical attachment level, and HbAlc served as strong predictors of gingival index, irrespective of gender. Similarly, a study on Korean T2DM patients (aged 40-70) showed HbAlc and fasting plasma glucose as strong predictors of periodontal health, irrespective of gender [21]. The presence of obesity and hyperglycaemia aggravates gingival inflammation while optimal adiposity and glycaemic control leads to a concomitant reduction in gingival inflammation [22]. Well defined studies have demonstrated that T2DM subjects with periodontitis are highly prone to diabetic nephropathy [23], diabetic retinopathy, and ketoacidosis than T2DM subjects without periodontitis [24]. In this context, our observations gain importance as gingival index and HbAlc can be used together for better monitoring of elderly T2DM patients for secondary complications of diabetes.

Considering clinical management for T2DM, we observed no significant differences between T2DM patients treated by oral metformin/sulphonylureas and T2DM patients on insulin. This may be due to the fact that this is an observational study and a prospective follow-up study is required to delineate the effects of medications on periodontal health. In addition, patients in our study were age and BMI matched with no overt microvascular and macrovascular complications during the course of the study. This could have resulted from an absence of differential effects, due to antihyperglycemic therapy. It would be interesting to observe the differential effects of 
TABLE 5: Gender specific correlations of clinical variables with gingival index.

\begin{tabular}{|c|c|c|c|c|}
\hline \multirow{2}{*}{ Variables } & \multicolumn{2}{|c|}{ Correlation coefficient } & \multicolumn{2}{|c|}{$p$ value } \\
\hline & Males & Females & Males & Females \\
\hline Age (years) & 0.30 & 0.05 & $<0.001$ & 0.60 \\
\hline BMI $\left(\mathrm{kg} / \mathrm{m}^{2}\right)$ & 0.27 & 0.02 & $<0.01$ & 0.77 \\
\hline HbAlc (\%) & 0.30 & 0.26 & $<0.001$ & $<0.01$ \\
\hline Pocket depth (mm) & 0.57 & 0.63 & $<0.001$ & $<0.001$ \\
\hline Clinical attachment level & 0.77 & 0.76 & $<0.001$ & $<0.001$ \\
\hline
\end{tabular}

$p<0.05$ : statistically significant.

TABLE 6: Gender specific predictors of gingival index in patients with T2DM.

\begin{tabular}{lcccccc}
\hline \multirow{2}{*}{$\begin{array}{l}\text { Predictor variables for gingival } \\
\text { index }\end{array}$} & \multicolumn{2}{c}{ Standardized coefficient (beta } & \multicolumn{2}{c}{ 95\% confidence interval } & \multicolumn{2}{c}{$p$ value } \\
& Males & Females & Males & Females & Males & Females \\
\hline BMI $\left(\mathrm{kg} / \mathrm{m}^{2}\right)$ & 0.06 & 0.00 & $0.03,08$ & $-0.02,0.02$ & $<0.001$ & 0.87 \\
HbAlc $(\%)$ & 0.006 & 0.03 & $0.03,0.04$ & $-0.02,0.92$ & 0.75 & 0.29 \\
Pocket depth $(\mathrm{mm})$ & 0.10 & 0.12 & $0.05,0.15$ & $0.05,0.19$ & $<0.001$ & $<0.001$ \\
Clinical attachment level & 0.24 & 0.21 & $0.19,0.29$ & $0.15,0.28$ & $<0.001$ & $<0.001$ \\
\hline
\end{tabular}

$p<0.05$ : statistically significant; regression coefficient value: $\left(r^{2}\right)=0.63$.

TABLE 7: Correlation of gingival index with clinical variables based on antihyperglycemic medications.

\begin{tabular}{lcccc}
\hline Variables & \multicolumn{1}{c}{ OADs } & \multicolumn{2}{c}{$\begin{array}{c}\text { Insulin } \\
p \text { value }\end{array}$} \\
\hline Fasting blood glucose & Correlation coefficient & $p$ value & Correlation coefficient & 0.13 \\
BMI $\left(\mathrm{kg} / \mathrm{m}^{2}\right)$ & 0.41 & $<0.05$ & 0.05 & 0.6 \\
HbAlc $(\%)$ & 0.05 & 0.76 & 0.21 & $<0.05$ \\
Triglycerides $(\mathrm{mg} / \mathrm{dl})$ & 0.05 & 0.78 & 0.06 & 0.35 \\
Pocket depth $(\mathrm{mm})$ & 0.47 & $<0.01$ & 0.57 & $<0.001$ \\
Clinical attachment level & 0.73 & $<0.01$ & 0.73 & $<0.001$ \\
\hline
\end{tabular}

$p<0.05$ : statistically significant.

locally delivered subgingival metformin therapy and oral metformin in Asian Indians with $\mathrm{T} 2 \mathrm{DM}$, as done in an earlier study elsewhere [25].

It is important to note that in patients with T2DM, social factors can significantly impact oral health status and access to dental care. A large epidemiological survey reported that people with lower incomes had poorer oral health status especially as the type of oral healthcare provided was rarely preventive [26]. Importantly in this study we observed that T2DM patients of both groups did not practise brushing before bed time and habitual dental flossing. Most of the subjects reported having a dental examination only during tooth ache or intensive gum bleeding. We also observed that the study subjects lacked awareness of the importance of dental health probably due to less emphasis on dental health in clinical care of T2DM. This is similar to the observations of another study which reported T2DM as an important risk factor for periodontitis [27]. Furthermore, most T2DM patients reported that their treating physicians did not emphasize the need for a dental checkup as in semiurban India and dental care is available only as a separate speciality and is not part of a comprehensive diabetes care regimen. In view of such scenarios, the predictors derived in our study hold robust predictive value $\left(r^{2}\right.$ value $\left.=0.67\right)$ and can be effectively applied for screening and prevention of periodontal complications. Further, considering the social scenario of the study population, it would be very ideal if physicians treating patients with T2DM also advise them on periodic dental examinations and provide accessible dental care at affordable costs. We acknowledge the limitations of this study as being cross-sectional and non-interventional in design. Also, the correlation of periodontitis with secondary complications of T2DM was not studied. In this context, prospective, large scale studies are required in different parts of India to validate the observations. Nevertheless, this study has reported three simple and important variables that predict the risk of severe periodontitis in middle aged, obese subjects with T2DM which can be applied in other populations of Tamil Nadu.

With ever increasing burden of T2DM in India, dental care and screening programs need to be scaled up with sustained treatment regimens at affordable costs. A comprehensive dental care program for populations of different 
socioeconomic strata needs to be envisaged. Interventional studies at low cost need to be conducted to ameliorate and manage dental complications in T2DM.

\section{Conclusion}

Glycosylated hemoglobin, pocket depth, and clinical attachment level can be used in comprehensive screening of T2DM patients for periodontitis and for initiation of preventive therapy.

\section{Conflicts of Interest}

The authors declare no conflicts of interest regarding this study or the decision to publish it.

\section{Authors' Contributions}

S. Jai Karthik and Shajith Anoop conceptualized and designed the study. S. Jai Karthik conducted the study. Shajith Anoop analysed the data, wrote the manuscript, and revised it. Suresh Kumar recruited T2DM patients for the study. M. V. Usha Rani offered suggestions and participated in the discussion of the manuscript.

\section{Acknowledgments}

The authors thank the subjects who participated in this study.

\section{References}

[1] A. Cheema, D. Adeloye, S. Sidhu, D. Sridhar, and K. Y. Chan, "Urbanization and prevalence of type 2 diabetes in Southern Asia: a systematic analysis," Journal of Global Health, vol. 4, no. 1, article 010404, 2014.

[2] A. Kautzky-Willer, J. Harreiter, and G. Pacini, "Sex and gender differences in risk, pathophysiology and complications of type 2 diabetes mellitus," Endocrine Reviews, vol. 37, no. 3, pp. 278-316, 2016.

[3] Y.-Y. Wu, E. Xiao, and D. T. Graves, "Diabetes mellitus related bone metabolism and periodontal disease," International Journal of Oral Science, vol. 7, no. 2, pp. 63-72, 2015.

[4] P. Han, D. Sun, and J. Yang, "Interaction between periodontitis and liver diseases," Biomedical Reports, vol. 5, no. 3, pp. 267-276, 2016.

[5] T. Dietrich, M. Jimenez, E. A. K. Kaye, P. S. Vokonas, and R. I. Garcia, "Age-dependent associations between chronic periodontitis/edentulism and risk of coronary heart disease," Circulation, vol. 117, no. 13, pp. 1668-1674, 2008.

[6] P. M. Preshaw, A. L. Alba, D. Herrera et al., "Periodontitis and diabetes: a two-way relationship," Diabetologia, vol. 55, no. 1, pp. 21-31, 2012.

[7] J. Hintao, R. Teanpaisan, V. Chongsuvivatwong, C. Ratarasan, and G. Dahlen, "The microbiological profiles of saliva, supragingival and subgingival plaque and dental caries in adults with and without type 2 diabetes mellitus," Oral microbiology and immunology, vol. 22, no. 3, pp. 175-181, 2007.

[8] H. G. Mohamed, S. B. Idris, M. F. Ahmed et al., "Influence of type 2 diabetes on local production of inflammatory molecules in adults with and without chronic periodontitis: a crosssectional study," BMC Oral Health, vol. 15, no. 1, article 86, 2015.

[9] A. Zizzi, G. Tirabassi, S. D. Aspriello, M. Piemontese, C. Rubini, and G. Lucarini, "Gingival advanced glycation endproducts in diabetes mellitus-associated chronic periodontitis: An immunohistochemical study," Journal of Periodontal Research, vol. 48, no. 3, pp. 293-301, 2013.

[10] P. K. Khanuja, S. C. Narula, R. Rajput, R. K. Sharma, and S. Tewari, "Association of periodontal disease with glycemic control in patients with type 2 diabetes in Indian population," Frontiers of Medicine, vol. 11, no. 1, pp. 110-119, 2017.

[11] R. T. Demmer, D. R. Jacobs, and M. Desvarieux, "Periodontal disease and incident type 2 diabetes: Results from the first national health and nutrition examination survey and its epidemiologic follow-up study," Diabetes Care, vol. 31, no. 7, pp. 1373-1379, 2008.

[12] S. Shajithanoop, T. Periyasamy, and M. U. Rani, "Demographic Variations Influence Obesity in a Semi-urban Cohort of Tamil Nadu, South India," Life Science Journal, vol. 4, no. 2, pp. 87-95, 2017.

[13] A. Misra, P. Chowbey, B. M. Makkar et al., "Consensus statement for diagnosis of obesity, abdominal obesity and the metabolic syndrome for Asian Indians and recommendations for physical activity, medical and surgical management," Journal of the Association of Physicians of India, vol. 57, no. 2, pp. 163170, 2009.

[14] “Third Report of the National Cholesterol Education Program (NCEP) Expert Panel on Detection, Evaluation and treatment of High Blood cholesterol in Adults(Adult Treatment Panel (III) Final report," Circulation, vol. 106, no. 25, pp. 3143-3421, 2002.

[15] "World Health Organization: Definition, Diagnosis and Classification of Diabetes Mellitus and Its Complications Report of a WHO Consultation. Part 1: Diagnosis and Classification of Diabetes Mellitus," Department of Non communicable Disease Surveillance, Geneva, Switzerland, 1999.

[16] R. Ramasamy, S. J. Vannucci, S. S. D. Yan, K. Herold, S. F. Yan, and A. M. Schmidt, "Advanced glycation end products and RAGE: a common thread in aging, diabetes, neurodegeneration, and inflammation," Glycobiology, vol. 15, no. 7, pp. 16R28R, 2005.

[17] V. S. Patil, V. P. Patil, N. Gokhale, A. Acharya, and P. Kangokar, "Chronic periodontitis in type 2 diabetes mellitus: Oxidative stress as a common factor in periodontal tissue injury," Journal of Clinical and Diagnostic Research, vol. 10, no. 4, pp. BC12BC16, 2016.

[18] A. Miyawaki, S. Toyokawa, K. Inoue, Y. Miyoshi, and Y. Kobayashi, "Self-reported periodontitis and incident type 2 diabetes among male workers from a 5-year follow-up to MY health up study," PLoS ONE, vol. 11, no. 4, article e0153464, 2016.

[19] I. B. Lamster, B. Cheng, S. Burkett, and E. Lalla, "Periodontal findings in individuals with newly identified pre-diabetes or diabetes mellitus," Journal of Clinical Periodontology, vol. 41, no. 11, pp. 1055-1060, 2014.

[20] P. Rajan, M. Nera, A. K. Pavalura, N. Medandrao, and S. C. Kumar, "Comparison of glycosylated hemoglobin (HbAlC) levels in patients with chronic periodontitis and healthy controls," Dental Research Journal (Isfahan), vol. 10, no. 3, pp. 389-393, 2013.

[21] E.-K. Kim, S. G. Lee, Y.-H. Choi et al., "Association between diabetes-related factors and clinical periodontal parameters in type-2 diabetes mellitus," BMC Oral Health, vol. 13, no. 1, article 64, 2013. 
[22] R. S. Levine, "Obesity, diabetes and periodontitis - A triangular relationship?” British Dental Journal, vol. 215, no. 1, pp. 35-39, 2013.

[23] W. A. Shultis, E. J. Weil, H. C. Looker et al., "Effect of periodontitis on overt nephropathy and end-stage renal disease in type 2 diabetes," Diabetes Care, vol. 30, no. 2, pp. 306-311, 2007.

[24] T. J. Orchard, K. Y.-Z. Forrest, D. Ellis, and D. J. Becker, "Cumulative glycemic exposure and microvascular complications in insulin-dependent diabetes mellitus: the glycemic threshold revisited," JAMA Internal Medicine, vol. 157, no. 16, pp. 18511856, 1997.

[25] A. R. Pradeep, N. S. Rao, S. B. Naik, and M. Kumari, "Efficacy of varying concentrations of subgingivally delivered metformin in the treatment of chronic periodontitis: A randomized controlled clinical trial," Journal of Periodontology, vol. 84, no. 2, pp. 212-220, 2013.

[26] S. Wamala, J. Merlo, and G. Boström, "Inequity in access to dental care services explains current socioeconomic disparities in oral health: The Swedish National Surveys of Public Health 2004-2005," Journal of Epidemiology and Community Health, vol. 60, no. 12, pp. 1027-1033, 2006.

[27] A. Aggarwal and S. R. Panat, "Oral health behavior and HbAlc in Indian adults with type 2 diabetes," Journal of Oral Science, vol. 54, no. 4, pp. 293-301, 2012. 


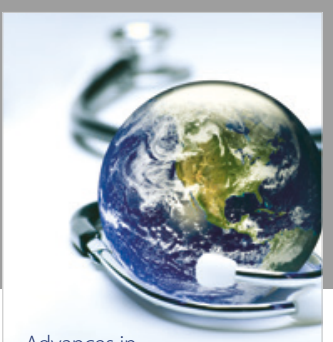

Advances in
Public Health

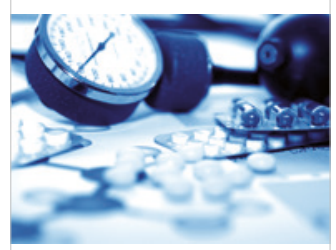

Case Reports in

Medicine

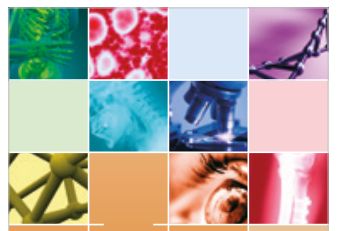

niernational Journal of

Biomaterials
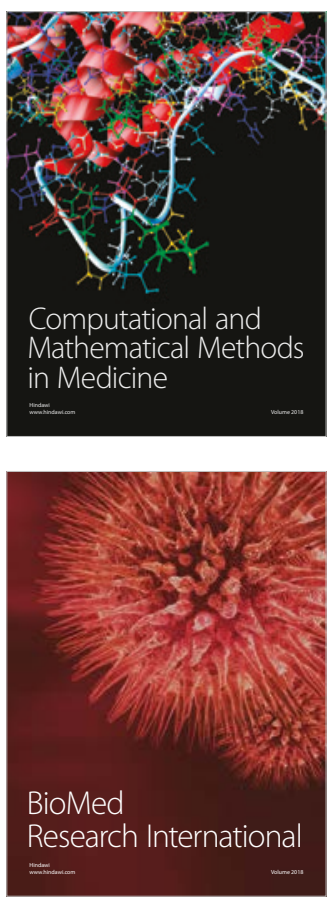

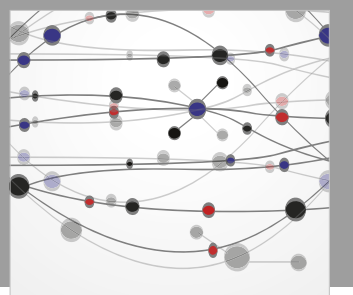

The Scientific World Journal Dentistry

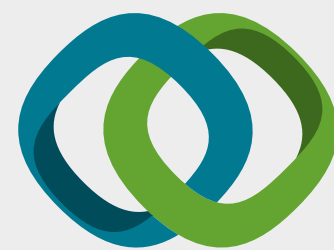

Hindawi

Submit your manuscripts at

www.hindawi.com
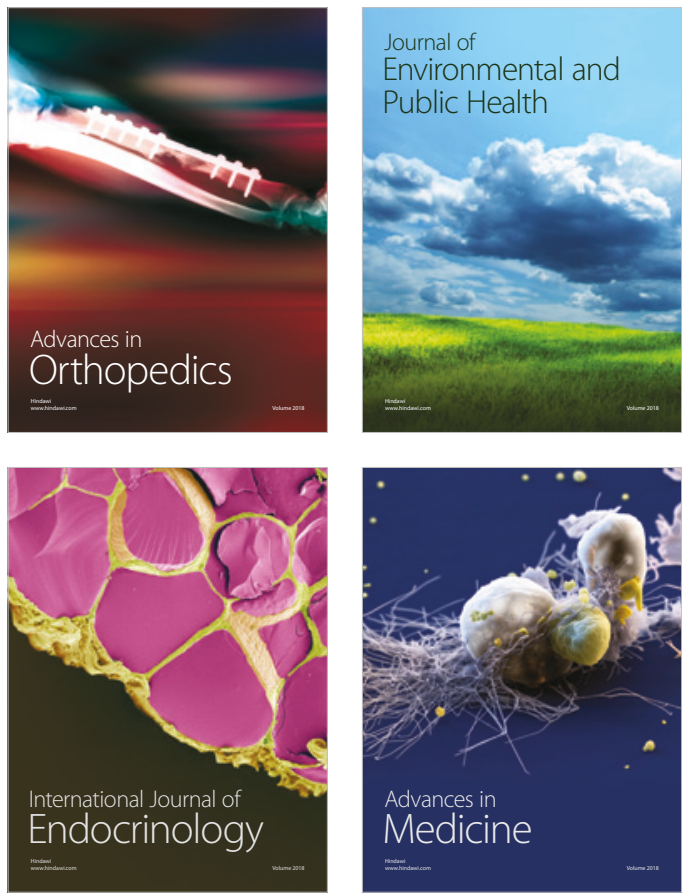
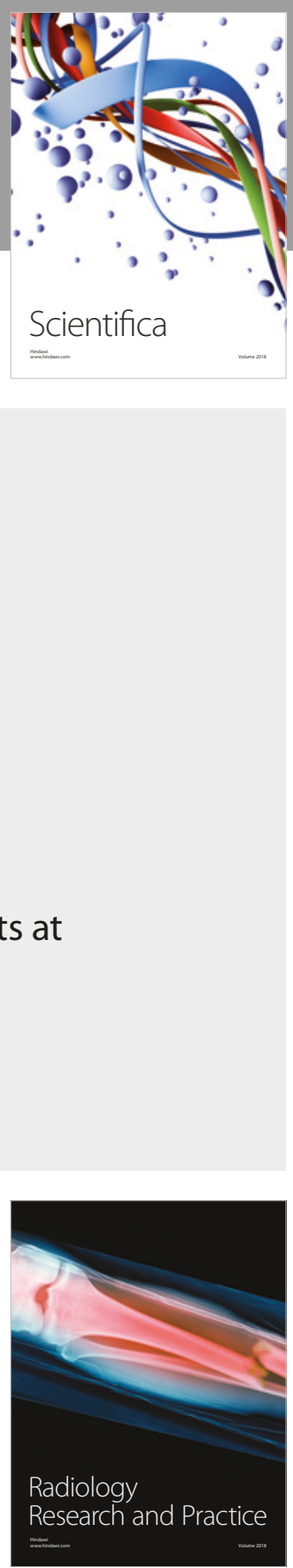

Scientifica

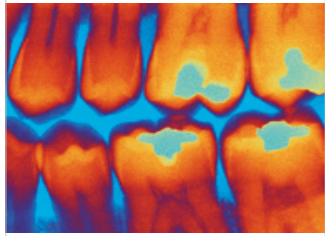

Case Reports in

Dentistry
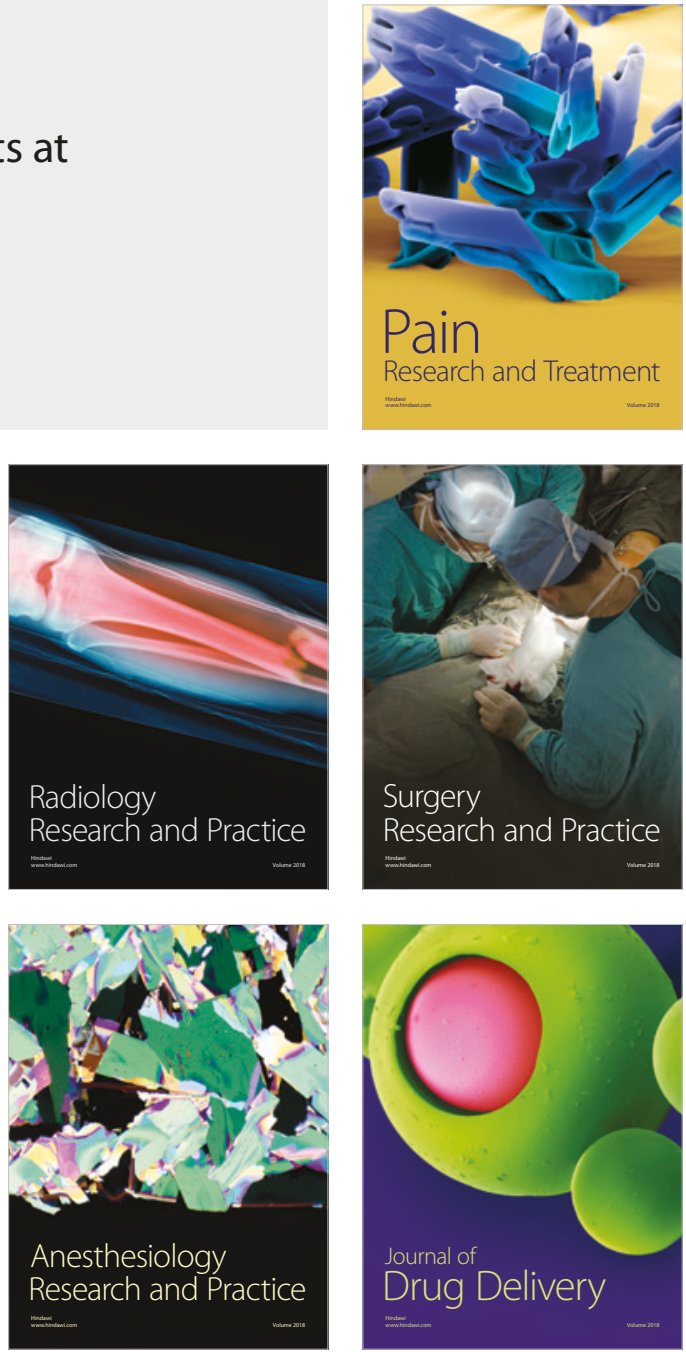